\title{
Physics model-informed Gaussian process for online optimization of particle accelerators
}

\author{
Adi Hanuka®, ${ }^{1, *}$ X. Huang $\odot,{ }^{1}$ J. Shtalenkova, ${ }^{1}$ D. Kennedy, ${ }^{1}$ A. Edelen, ${ }^{1}$ Z. Zhang, ${ }^{1}$ \\ V. R. Lalchand, ${ }^{2}$ D. Ratner, ${ }^{1}$ and J. Duris ${ }^{1}$ \\ ${ }^{1}$ SLAC National Accelerator Laboratory, Menlo Park, California 94025, USA \\ ${ }^{2}$ University of Cambridge, The Old Schools, Cambridge, CB2 ITN, United Kingdom
}

(Received 15 March 2021; accepted 2 June 2021; published 8 July 2021)

\begin{abstract}
High-dimensional optimization is a critical challenge for operating large-scale scientific facilities. We apply a physics-informed Gaussian process (GP) optimizer to tune a complex system. Typical GP models learn from past observations to make predictions, but this reduces their applicability to systems where there is limited relevant archive data. Instead, here we use a fast approximate model from physics simulations to design the GP model. The GP is then employed to make inferences from sequential online observations in order to optimize the system. Simulation and experimental studies were carried out to demonstrate the method for online control of a storage ring. Our method is a simple prescription to construct a custom GP model, including correlations between the high-dimensional input space, while encoding the physical response of a system. The ability to inform the machine-learning model with physics, without relying on the availability and range of prior data, may have wide applications in science.
\end{abstract}

DOI: 10.1103/PhysRevAccelBeams.24.072802

\section{INTRODUCTION}

Online control and tuning of scientific experiments and facilities, such as free electron lasers and storage ring light sources, are challenging tasks, since those systems often consist of hundreds of correlated parameters that could be adjusted in order to find a set of parameter values to achieve optimal target performance. Automated tuning can help deliver the highest beam quality to scientific users during operation and reduce tuning time for operation mode switching. This would be enabled by efficient online optimization algorithms, which are necessary in particle accelerators because, although physics models exist, there are often significant differences between the simulation and the real accelerator. The critical requirement for a suitable tuning algorithm is the ability to find the optimum in a complex parameter space both robustly and with high efficiency (minimum number of steps).

Traditional model-independent optimization methods, that do not require the gradient of the system, such as the standard optimizer Nelder-Mead simplex [1], may not work well for online applications when the target is noisy. Other local, model-independent methods, such as robust

\footnotetext{
Corresponding author. adiha@slac.stanford.edu

Published by the American Physical Society under the terms of the Creative Commons Attribution 4.0 International license. Further distribution of this work must maintain attribution to the author(s) and the published article's title, journal citation, and DOI.
}

conjugate direction search (RCDS) [2,3] which uses a parabolic fit of points, offer resilience to noise by taking a large number of samples, thereby often taking a long time to converge or require initial conditions closer to the optimum [4]. Machine learning model-based optimization methods may be beneficial to improve the quality of the solution, the speed of convergence and robustness to noise.

Machine learning model-based methods for online optimization typically rely on learning from previously observed data. However, limited sparse sampling of high dimensional archived data may be insufficient, for example when learning correlations between various control variables. In addition, learning from archive data becomes impossible when preparing for configurations where there is insufficient relevant experimental data or it does not exist. On the other hand, approximate physics models cannot be applied directly on the system to be optimized. Those need to be calibrated and even then cannot exactly fit the observed data. In this paper we circumvent the limitations of both approaches by approximating the covariance of the system directly from the physics model and then building a model from a few online observations. Physics models may capture the qualitative response of the objective with respect to controls better than archive data. Incorporating those into machine learning models may increase the speed of convergence and robustness of an online tuning process.

Bayesian optimization is a model-based approach to optimizing expensive to evaluate, black-box systems with possibly noisy inputs and outputs [5-7]. Online tuning by 
Bayesian optimization involves two main components: (i) online surrogate model $g(x)$, i.e., a fast-executing mathematical model approximating the objective $f(x)$ response to a vector of input control values $x$. This model is iteratively updated with observed data during optimization. (ii) An acquisition function which suggests the next observation to make based on the current surrogate model built from the observed data so far. A Gaussian process (GP) [8] is a popular choice for the surrogate model since it provides not only a prediction of the system's response but the uncertainty in that prediction as well. GPs predict a distribution of possible functions compatible with observations by utilizing a covariance function, called the kernel, describing relationships between those observations. The flexibility to capture the complex dependencies encountered in modern experiments lies in the design of this kernel. Learning the kernel function rather than the objective function itself is less prone to errors resulting from dependencies on drifts or random hidden variables.

Bayesian optimization with Gaussian process surrogate models has been increasingly applied to a range of fields; for example, materials design [9], pharmaceutical product development [10], experimental control [11], medical physics [12], nuclear physics [13], and more recently, it has been successfully demonstrated on linear accelerators [14-19]. Most of this line of work contains a Gaussian process with diagonal kernels (without correlations) learned from archive data. In Ref. [16], we learned correlations from a physics model and combined them with a diagonal kernel learned from archived machine data. However, often archive data is limited or insufficient to learn a kernel which captures the qualitative system's response. Even then this empirical kernel selection is computationally expensive. Therefore, the ability to easily learn the kernel directly from a physics model would turn GPs into a practical tool applicable for optimizing physical systems and configurations with limited data or even without any archived data.

In this paper we experimentally demonstrate a physicsinformed Gaussian process, where we use a physical model to directly derive the GP kernel including correlations. As an alternative to the traditional empirical kernel learning procedure using prior data [8], we construct the kernel from the physical model's basis-functions. The basis-function kernel eliminates the need for many data samples (either observed or simulated) and the traditional empirical kernel selection. As our primary result, we demonstrate experimentally the physics-informed basis-function approach effectiveness by comparing performance with the traditional approach and several other algorithms on the SPEAR3 storage ring facility [20] for minimizing the vertical emittance with respect to 13 skew quadrupole magnets. We finally discuss the importance of constructing a kernel that is representative of the system to be modeled.

\section{METHODS}

A Gaussian process (GP) is defined using mean and covariance functions $g(x) \sim \mathcal{G P}\left(m(x), k\left(x_{\mathrm{i}}, x_{\mathrm{j}}\right)\right)$, where $x_{\mathrm{i}}, x_{\mathrm{j}}$ are all possible pairs in the input domain. The mean function $m(x)$ describes the expected value of the objective, and the kernel $k\left(x_{\mathrm{i}}, x_{\mathrm{j}}\right)$ characterizes similarities between possible objective function values at different input points $x_{\mathrm{i}}$ and $x_{\mathrm{j}}$. While the optimum of the objective function may fluctuate day to day, the kernel captures the underlying behavior, allowing it to well represent the function given sampled data. To account for the observations' noise, we model the noise as independent and identically distributed Gaussian random variables with a mean of zero and a variance of $\sigma_{n}^{2}$. The corresponding Gaussian noise kernel is $k_{\text {noise }}\left(x_{\mathrm{i}}, x_{\mathrm{j}}\right)=\sigma_{n}^{2} \delta_{\mathrm{i}, \mathrm{j}}$, where $\delta$ is the Kronecker delta function. The GP is constructed directly from sampled instances, thus allowing the model's complexity to grow with observations and adapt to previously unexplored regions of the input space.

One of the critical steps in achieving an operational GP optimizer for complex systems is constructing a kernel which encodes the underlying behavior and relationships in the modeled data. For systems with high-dimensional data structures, we can create kernels which are capable of representing more complicated relationships between parameters from simpler ones by adding or multiplying kernels [21,22] or applying a nonlinear transform to the input data [23-25]. In principle, general properties of kernels are controlled by a number of hyperparameters. Usually, kernels and their hyperparameters are chosen through maximizing the probability of the data given the Gaussian process model (this technique is referred to as ML-II) [8]; see the Appendix A for more details on this approach. When using experimental archived data we refer to this approach as a data-informed ML-II Gaussian process. In order to capture the system's response to controls this method requires sufficient appropriate data points to cover the full parameter space. However, in many cases the data is sparsely sampled, especially in highdimensional systems. Time constraints for gathering data in new configurations or preliminary commissioned machines further limit sample density.

As an alternative, physics simulations could be used instead of experimental data [26,27], making it possible to learn a kernel if there is only little or even no historical data at all. We refer to this approach to kernel construction as physics-informed. However, as in the data-informed case, care must be taken in sampling the simulation input space to capture the objective's complexity as well as correlations between the input parameters. Using simulation data can be an expensive process and may require long computational times since a high dimensional input space would require many evaluations of a possibly slow simulation. In this case, using ML-II is costly, since the computational 
complexity scales cubically with the number of points. Therefore, there is a need to develop methods to find the best kernel and its hyperparameters without relying on many data samples (either observed or simulated), while allowing for the incorporation of prior physics knowledge. This would also increase the kernel's interpretability and may help gain real insight into the system.

In order to address this need, we calculate the kernel directly from a physical model. In general, there is a growing interest in incorporating knowledge of the physical system to optimize into machine learning models. For example, previous studies used governing partial differential equations to numerically calculate the covariance matrices [28-30]. In this work, we leverage the connection between infinitely wide Bayesian neural networks and Gaussian processes, to calculate the covariance function from an explicit basis function [8,31,32]:

$$
k\left(x_{\mathrm{i}}, x_{\mathrm{j}}\right) \propto \int_{-\infty}^{\infty} \phi\left(x_{\mathrm{i}}-c\right) \phi\left(x_{\mathrm{j}}-c\right) d c
$$

where $c$ denotes the center of the basis function $\phi(x)$. We refer to GPs based on kernels designed in this way as basisfunction GPs. Alternatively if the power spectral density of the system is easier to model, the covariance function can be calculated from the amplitude of the Fourier transform of the power spectral density [33].

For example, a radial basis function of the form $\phi(x)=\exp \left(-x^{\mathrm{T}} \Sigma x / 2\right)$, results in the radial basis-function kernel $k\left(x_{\mathrm{i}}, x_{\mathrm{j}}\right) \propto \exp \left(-\left(x_{\mathrm{i}}-x_{\mathrm{j}}\right)^{T}(\Sigma / 2)\left(x_{\mathrm{i}}-x_{\mathrm{i}}\right) / 2\right)[8,31]$, where $\Sigma$ is the precision matrix, and (.. $)^{T}$ is the transpose operation. This type of basis-function is useful for modeling many smooth functions. The precision matrix is a symmetric matrix encoding properties of the function. For example, if there are no correlations between input parameters, $\Sigma=\operatorname{diag}(l)^{-2}$ is a diagonal matrix wherein $l$ is a vector of characteristic lengthscales. The latter specifies how function values at two points separated in space along a single dimension (for example, a quadrupole magnet strength) relate to each other.

In what follows, we use an approximation to the physics model as the basis-function to design the kernel using Eq. (1). This allows the GP to make predictions of the system using the covariance of the physics model as an estimate of that of the system, rather than learning the kernel from data. We refer to this approach as physicsinformed basis-function GP. Learning the kernel from simulated data instead of machine data is a form of kernel transfer learning $[34,35]$. Furthermore, constructing the kernel from basis-functions without using the likelihood function is a form of Gaussian processes with likelihoodfree inference [36,37].

In this paper we consider the task of finding the peak of a system, which has a physics model of sufficient fidelity to capture the qualitative system's response [38]. For example, the simulation could have an unknown scaling and translation with respect to the machine but have a similar functional form. In order to calculate the physics model's basis function, in this work, we consider systems that can be roughly approximated with a Gaussian around the optimum of the simulation. The simulation's optimal point is assumed to be known thanks to a priori understanding of the underlying model of the user's specific problem. We then approximate the basis function by expanding the log of the simulation $\hat{f}(x)$ about a point $x_{0}$ close to the global optimum with an analytic expansion to second order, after subtracting off the asymptotic behavior $\hat{f}(\infty)>0$. By expanding the log of the simulation to the second order we assure that the expansion would be a Gaussian with a relatively good fit also far from the optimum.

We build the basis function by evaluating the gradient $(G)$ and the Hessian $(H)$ of the log of the simulation $G_{\mathrm{i}}=$ $\left.\partial_{x_{\mathrm{i}}} \log [\hat{f}(x)-\hat{f}(\infty)]\right|_{x=x_{0}}, H_{\mathrm{i}, \mathrm{j}}=\left.\partial_{x_{\mathrm{i}}} \partial_{x_{\mathrm{j}}} \log [\hat{f}(x)-\hat{f}(\infty)]\right|_{x=x_{0}}$ via numerical differentiation. The resulting expansion is $\log \left[\hat{f}\left(x_{0}\right)-\hat{f}(\infty)\right]+\left(x-x_{0}\right)^{\mathrm{T}} G+\frac{1}{2}\left(x-x_{0}\right)^{\mathrm{T}} H\left(x-x_{0}\right)$. If the expansion point $x_{0}$ is an optimum in the simulation as in the work presented here, then the gradient may be neglected, and the basis function has the functional form of a Gaussian:

$\phi(x)=\left[\hat{f}\left(x_{0}\right)-\hat{f}(\infty)\right] \exp \left[\frac{1}{2}\left(x-x_{0}\right)^{\mathrm{T}} H\left(x-x_{0}\right)\right]$.

Then Eq. (2) is used to calculate the associated covariance function by applying Eq. (1). The resulting covariance function has the same functional form as the radial basisfunction kernel [8], with a precision matrix half that of the Hessian above $\Sigma=-H / 2$. The function value $\hat{f}(\infty)$ was taken into account as the GP prior mean.

\section{EXPERIMENT}

In what follows we demonstrate experimentally the effectiveness of the physics-informed basis-function approach on SPEAR3 [20], a third generation storage ring light source operating with low emittance, which results in high photon beam brightness. The goal of this optimization task is to minimize the average vertical emittance with skew quadrupoles. Similar types of optimization tasks are ubiquitous in particle accelerators. While there are cases where simultaneous optimization of multiple objectives is required [39], many of the practical tuning problems are single objective ones $[16,40]$. Furthermore, in many cases, multiple objectives could be combined into a single objective by combining them with weights relative to each other.

In an ideal electron storage ring, the vertical emittance is nearly zero. However, in reality there are various sources of errors that give rise to a finite vertical emittance, such as vertical dispersion in dipole magnets and linear betatron coupling between horizontal and vertical planes. Those error sources can be compensated by skew quadrupole 


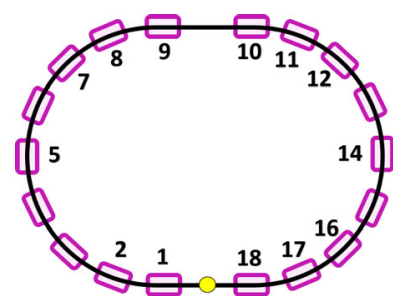

(a) Layout of SPEAR3

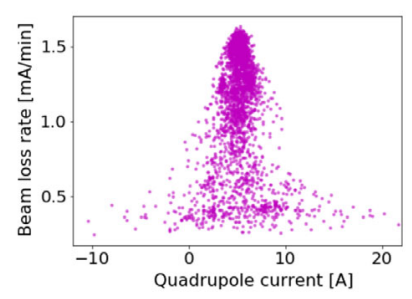

(b) Beam loss rate
FIG. 1. (a) Layout of the SPEAR3 storage ring with the 13 free skew quadrupoles used for the online optimization of beam loss rate. The non-destructive current monitor is shown as a yellow dot. (b) Beam loss rate projected on a single skew quadrupole current. The data is taken from archived operations scans.

magnets. In SPEAR3, there are 13 free skew quadrupoles for vertical emittance control (they do not change the horizontal emittance)—see Fig. 1(a).

While the projected vertical emittance can be measured at one location in the ring, the single measured value does not represent the average vertical emittance as the vertical emittance typically varies with location. The average vertical emittance can be represented with an indirect measure, the high-current beam loss rate, which is nondisruptively monitored. When the beam loss is dominated by Touschek scattering, as is the case for a low emittance, high-current beam, the beam loss rate is inversely proportional to the volume of the beam bunch. Since skew quadrupoles do not change the longitudinal beam size, and, for the low-level coupling case, they do not change the horizontal beam size, either, changes of the beam loss rate directly reflect the changes of the vertical beam size. Since the latter is proportional to the square root of the vertical emittance, minimizing vertical emittance is equivalent to maximizing beam loss rate (Amperes per minute) with skew quadrupoles magnets [40].

In order to build the GP kernel to be used in the optimization using Eq. (1), we calculated the Hessian at the maximum beam loss rate point (see Fig. 1(b)) in two ways. First, we used a fast-executing neural-network model trained on the SPEAR3 storage simulation data, obtained with the Accelerator Toolbox [3] (details described in the Appendix B). This facilitates fast calculation of the Hessian. Second, we numerically calculated the Hessian directly from a noiseless SPEAR3 simulation. We found these to be in acceptable agreement.

The precision matrix of the kernel containing both lengthscales and correlations was calculated from physics simulations. The kernel's amplitude was evaluated from the variance of a uniform distribution spanning the objective's range, which was similar to the value obtained by the MLII, using data from dedicated raster scans of the machine. The kernel's noise was measured from a few live machine measurements $\left(\sigma_{n}=0.04\right)$. This noise is constant and is not correlated with the loss rate value.
The skew quadrupole magnet currents were limited to the search range of $[-20,20]$ Amperes and were set to zero before scanning each time, and this reduced the beam loss rate to $\hat{f}(\infty) \sim 0.5 \mathrm{~mA} / \mathrm{min}$. The loss rate was evaluated by computing the change in the beam current loss rate over one second. Then we waited one second to let the quadrupoles current settle in the next point. For each set of experiments, the GP optimizer was initialized with a kernel, prior mean of $\hat{f}(\infty)$, and first observed point.

\section{RESULTS AND DISCUSSION}

In what follows, we show that online optimization using the GP optimizers surpasses the current established optimization algorithms, Nelder-Mead simplex [1] and robust conjugate direction search (RCDS) [2], which are routinely used to tune particle accelerator systems [41]. All algorithms were tested on the same computational hardware under similar loading conditions. Since the basis-function approach makes it easier to calculate correlations, it is feasible to create a kernel including off-diagonal elements, whereas for the available data, the ML-II approach is limited to resolving a diagonal-only kernel. The physicsinformed basis-function approach converged faster than the traditional data-informed ML-II approach, and the gain in speed from including correlations is expected to increase with higher input dimension [16].

Figure 2(a) shows results from online optimization of the beam loss rate simultaneously on 13 skew quadrupole magnets. The Gaussian process (GP) optimizer with a physics-informed basis-function kernel reached an optimum of $1.67 \mathrm{~mA} / \mathrm{min}$ in the smallest number of function evaluations (30 to 40 steps which are equivalent to 0.5 to 1 minutes). The archive data-informed ML-II GP achieved $1.62 \mathrm{~mA} / \mathrm{min}$ in 40 to 60 steps (0.66 to 1.2 minutes). The Nelder-Mead simplex optimizer achieved on $1.32 \mathrm{~mA} /$ min in approximately 160 steps (2.6 minutes). The RCDS optimizer achieved $1.66 \mathrm{~mA} / \mathrm{min}$, but took longer to converge; approximately 180 steps wherein each step is 6 seconds-total of 20 minutes. This increased measurement step time for RCDS allows for a reduced measurement noise of $0.02 \mathrm{~mA} / \mathrm{min}$ which was found helpful for RCDS to converge. In contrast, the GP optimizers handle the noisier measurements better, resulting in shorter step times. We also found that these results were consistent with subsequent tests where each optimizer started from the same random starting point with the same initial beam loss rate as before.

A comparison of the above optimizers in simulation environment is shown in Fig. 2(b). Although the SPEAR3 simulator does not capture the full complexity of the live machine, it allows us to compare the relative performance of the optimizers with a simulated objective function, which we find consistent with the online optimization. In simulation, on average, the physics-informed basis- 


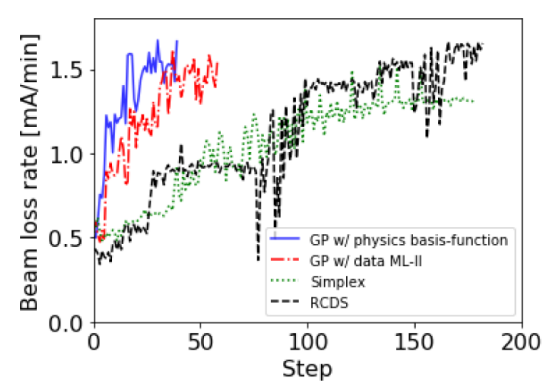

(a) Online machine optimization Comparison of optimizers

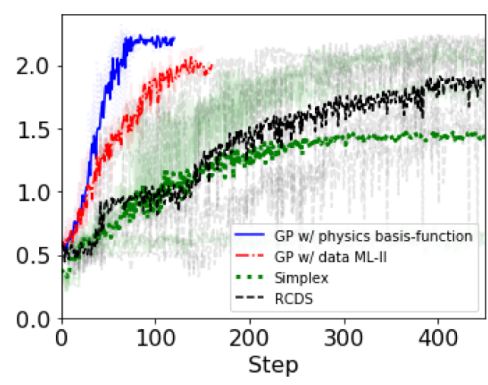

(b) Simulated optimization Comparison of optimizers

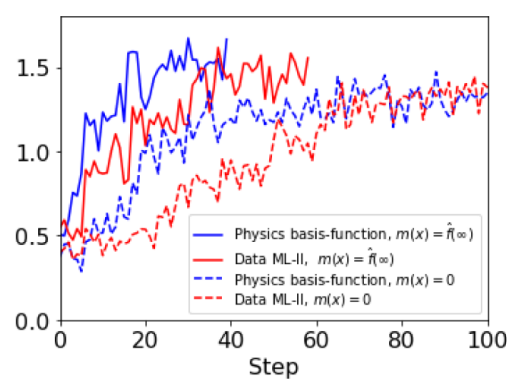

(c) Online machine optimization Prior mean effect

FIG. 2. (a) Comparison of optimization of beam loss rate over 13 skew quadrupole magnets for Gaussian process (GP) with physicsinformed kernel including off-diagonal elements (blue), GP with diagonal-only data-informed kernel (red), Nelder-Mead simplex (green), and RCDS (black). Each step corresponds to approximately 1 to 2 seconds for GP and simplex, and to 6 seconds for RCDS. (b) Simulations using the conditions of (a). Six individual scans for each method, with means shown by thick lines, are consistent with the relative performance of the online optimizations. (c) Comparison of GP optimizers with the objective's offset as prior mean (solid), and without (dashed).

function approach finds a better optimum in fewer iterations than the other methods. In addition, the spread of six individual scans for each method (with means in thick lines) reveals the robustness of the GPs, which follow similar trajectories for individual scans.

Notably, the maximum available value of the simulated objective function is higher than the corresponding online optimization value. This is understandable as the actual machine has more coupling error sources than modeled in the simulation. In reality, on the machine we cannot expect more than $\sim 1.7 \mathrm{~mA} /$ min with the experimental conditions used in this paper. Nevertheless, the simulation is adequate to capture the qualitative objective response with respect to skew quadrupoles in order to evaluate the physics-informed covariance function.

Our primary goal was to minimize the vertical emittance. In order to verify that, we derived the emittance ratio between the vertical and horizontal planes (show in parentheses) from the orbit response matrix [42] at three quadrupoles settings: the initial setting with 13 skew quads set to zero $(0.71 \%)$, the normal operation lattice $(0.05 \%)$, and the GP optimized solution $(0.032 \%)$ respectively. Based on these combined simulation and experimental results, we expect the physics-informed basis-function approach to be the most effective in practice.

Constructing a Gaussian process model that is representative of the system is a crucial step for increasing the effectiveness of the optimization. So far, we showed the effect of the kernel by comparing two GPs with a prior mean equal to the objective offset $(m(x)=\hat{f}(\infty))$. Next, we demonstrate the effect of the prior mean by comparing a GP model with a prior mean to one without $(m(x)=0)$. Figure 2(c) shows a comparison of the optimization results of the GP approaches with $m(x)=0$ and $\hat{f}(\infty)$. For both cases, the GP optimizers with zero prior mean (dashed lines) converged slower to a lower optimum, further validating the importance of the prior mean choice. In addition, the physics-informed basis-function GP optimizer converged faster than the data-informed ML-II GP optimizer in both cases.

\section{CONCLUSION}

We presented and experimentally demonstrated a method incorporating physics models directly into a Gaussian process (GP) optimizer. Our method presents a simple and fast way to construct the GP kernel, including correlations between devices, without relying on the availability and range of prior data samples. The physics-informed GP, which is more representative of the system, performed faster in an online optimization task compared to routinely used optimizers.

This method could be easily adapted to other physical systems which have a rough model, simulations or theory that could be approximated with a Gaussian. The method is particularly well suited to analytical or differentiable models [43], as well as surrogate models [44]. It would also be applicable for automatic tuning and control of machines and other complex configurations where historical data is unavailable or insufficient to resolve the kernel's hyperparameters, including correlations. The incorporation of prior physics knowledge would increase the attractiveness of Bayesian optimization with GPs for practitioners across various scientific domains.

\section{ACKNOWLEDGMENTS}

The authors are grateful to the SPEAR3 operators and engineers for their help with live tests on the storage ring, and to Michael Kagan and Stefano Ermon for fruitful discussions. This work was supported by the Department of Energy, Laboratory Directed Research and Development program at SLAC National Accelerator Laboratory, under 
Contract No. DE-AC02-76SF00515, by Office of Basic Energy Sciences under Grant No. FWP 100637, and by Office of Advanced Scientific Computing Research under Grant No. FWP 2018-SLAC-100469ASCR.

\section{APPENDIX A: ML-II KERNEL LEARNING PROCEDURE}

In this Appendix we first describe the ML-II kernel learning, namely how we calculated the kernel's hyperparameters from data. Second, we show experimental comparison of the data-informed Gaussian process for various kernels. Lastly, we compare the performance in simulated optimization of the physics-informed kernel learned by either ML-II or basis-function procedure to the data-informed kernel learned by ML-II.

The ML-II kernel learning procedure entails learning the hyperparameters of the chosen kernel family using historical data. If the historical data is an archive of machine data, we refer to the approach as data-informed ML-II Gaussian Process, whereas if simulated data is used, we refer to the approach as physics-informed ML-II Gaussian process. Figure 1(b) in the paper shows a typical response of the beam loss rate with respect to a skew quadrupole magnet. The Gaussian-like shape of this response could either be modeled with the popular radial basis function (RBF) kernel used for smooth sample functions, or with the Matern kernels [see Eq. (A2)] usually used for much rougher sample functions. Given two inputs $x_{\mathrm{i}}, x_{\mathrm{j}}$, the functional form of the RBF kernel is

$$
k_{\mathrm{RBF}}\left(x_{\mathrm{i}}, x_{\mathrm{j}}\right)=\sigma_{f}^{2} \exp \left(-\frac{1}{2} \Delta_{x}^{\mathrm{T}} \Sigma \Delta_{x}\right),
$$

where $\sigma_{f}^{2}$ is the kernel's amplitude variance, $\Sigma$ is the precision matrix, $\Delta_{x}=x_{\mathrm{i}}-x_{\mathrm{j}}$, and $(. .)^{\mathrm{T}}$ denotes a matrix transpose operation. The functional forms of the Matern32 and Matern52 kernels are

$$
\begin{gathered}
k_{\mathrm{M} 32}=\sigma_{f}^{2}\left(1+\sqrt{3 \Sigma^{-1}} \Delta_{x}\right) \exp \left(-\sqrt{3 \Sigma} \Delta_{x}\right) . \\
k_{\mathrm{M} 52}=\sigma_{f}^{2}\left(1+\sqrt{5 \Sigma} \Delta_{x}+\frac{5}{3} \Delta_{x}^{\mathrm{T}} \Sigma \Delta_{x}\right) \exp \left(-\sqrt{5 \Sigma} \Delta_{x}\right) .
\end{gathered}
$$

In principle, the diagonal elements of the precision matrix $\Sigma$ are the inverse squared lengthscales, which specify how function values at two points separated in space along a single dimension (for example, a quadrupole magnet strength) relate to each other. The off-diagonal elements encode correlations between different dimensions (in this example, different quadrupoles). In the SPEAR3 case, due to the sparsity of the data, we could not resolve the correlations from data; thus, the precision matrix for the data-informed approach is a diagonal matrix.
To account for the observations' noise, we model the noise as independent and identically distributed Gaussian random variables with a zero mean and a variance of $\sigma_{n}^{2}$. The corresponding Gaussian noise kernel is $k_{\text {noise }}\left(x_{\mathrm{i}}, x_{\mathrm{j}}\right)=\sigma_{n}^{2} \delta_{\mathrm{i}, \mathrm{j}}$. As shown in Fig. 1(b) in the paper, the beam loss rate has an offset of $\sim 0.5$, which corresponds to the gas scattering background, beam losses due to collisions of the electron beam and the residual gas in the vacuum chamber. We accounted for the observation's offset as the GP prior mean.

Given a finite set $(\mathrm{X}, \mathrm{Y})$ of $M$ sampled data points, where $\mathrm{X}=\left\{x_{1}, \ldots, x_{M}\right\}$, each $x$ is of dimension $D$, $\mathrm{Y}=\left\{y_{1}, \ldots, y_{M}\right\}, y_{i}=f\left(x_{i}\right)+\sigma_{n}, i=1 \ldots M$ and $\sigma_{n}$ is the measurement's noise, we will find a set of kernel's hyperparameters $\theta=\left\{\sigma_{f}^{2}, \Sigma, \sigma_{n}^{2}\right\}$ that best describes the data. We maximize the log marginal likelihood [8] for the sampled data given by

$\log p(\mathrm{Y} \mid \mathrm{X}, \theta)=-\frac{1}{2} \mathrm{Y}^{\mathrm{T}} \mathbf{K}_{\mathrm{Y}}{ }^{-1} \mathrm{Y}-\frac{1}{2} \log \left|\mathbf{K}_{\mathrm{Y}}\right|-\frac{M}{2} \log 2 \pi$,

where $\mathrm{K}_{\mathrm{Y}}=\mathrm{K}_{\mathrm{XX}}+\sigma_{n}^{2} \mathrm{I}, \mathrm{K}_{\mathrm{XX}}=k(\mathrm{X}, \mathrm{X})$ is the covariance matrix, $\mathbf{I}$ is the identity matrix. The marginal likelihood quantifies the probability of $\mathrm{Y}$ given the GP kernel hyperparameters $(\theta)$; the first term accounts for the data fit and the second term accounts for a complexity penalty favoring simpler models which counteracts against overfitting. The third term is constant given a finite set of data points. This procedure of learning point estimates for hyperparameters through the maximization of the marginal likelihood is called type-II maximum likelihood (ML-II) [8]. We repeat this process for each kernel in Eqs. (A1), (A2) given archive machine data (raster scans of the quads). The resulting precision matrix for all kernels is diagonal, i.e., does not include correlations. Figure 3 compares online optimization on SPEAR3 using data-informed ML-II Matern kernels and the RBF kernel. Evidently, the Matern52 performed similar to RBF kernel, whereas the Matern32 converged on a lower optimum.

Lastly, in Fig. 4 we show a comparison of the simulated optimization performance with RBF kernel whose hyperparameters were learned in three different ways: ML-II on archived machine raster scans (red curve), ML-II on simulated raster scans (cyan curve), and basis function on simulation (blue curve). Since the ML-II procedure using raster scans enables resolving only the lengthscales, the resulting precision matrices are diagonal, whereas the basis-function procedure yield the correlations as well. Therefore, we also compare the performance of the kernel from the basis-function excluding the off diagonal elements (magenta curve). Notably, the models with diagonal kernels perform similarly. 


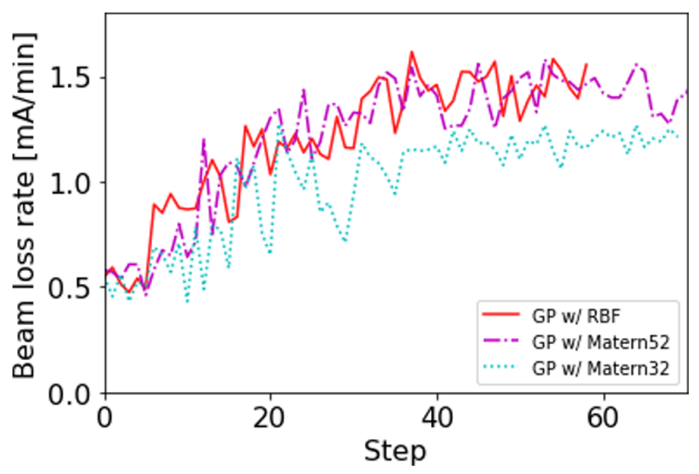

FIG. 3. Online machine optimization using RBF and Matern kernels for the archive data-informed Gaussian process with MLII kernel learning.

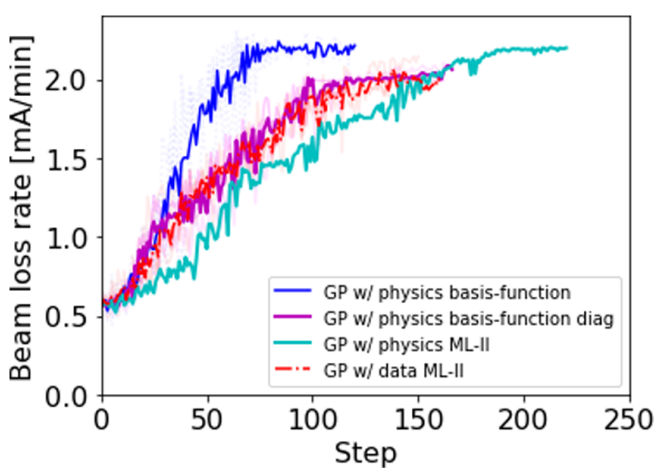

FIG. 4. Simulated optimization using RBF kernel for the datainformed Gaussian process with ML-II kernel learning (red), physics-informed Gaussian process with ML-II (cyan), and physics-informed Gaussian process basis-function, either with full kernel including correlations (blue), or diagonal kernel (magenta). The ML-II kernels are diagonal as well.

\section{APPENDIX B: SPEAR3 NEURAL NETWORK- BASED SURROGATE MODEL}

In this Appendix we describe the SPEAR3 surrogate model that was used for fast calculation of the physics model's Hessian. Global surrogate models are powerful fast-executing tools that are trained using sparse samples of a physics simulation [44]. These models are increasingly being adopted by the accelerator community to aid fast online simulation, offline experiment planning, and design studies. In this work, we used a deep neural network (NN) to approximate the SPEAR3 simulation. The NN had 6 hidden layers (with 90-100-100-100-80-70 nodes respectively) and tanh activation functions. Smaller networks were examined and did not predict the performance as well. The data set consisted of 85,263 samples across a broad range of the 13 magnet settings, along with corresponding beam loss rates. This data was split in proportions of 60-20-20 into training, validation, and testing data.
The NN-based surrogate model provides an attractive way to obtain high dimensional non-linear interpolation. We then calculated the Hessian of the log of the interpolation after subtracting the pedestal. The NN training was performed with Keras [45] and tensorflow [46] libraries, and the Hessian was calculated using the numdifftools python library [47]. Although we calculated the Hessian numerically in this case, building a NN with software that supports auto-differentiation also enables the Hessian to be calculated directly.

[1] J. A. Nelder and R. Mead, Comput. J. 7, 308 (1965).

[2] X. Huang, Phys. Rev. Accel. Beams 21, 104601 (2018).

[3] A. Terebilo et al., Accelerator modeling with Matlab Accelerator Toolbox, Proceedings of PAC2001, Chicago, IL (2001), pp. 3203-3205.

[4] A. Scheinker, A. Edelen, D. Bohler, C. Emma, and A. Lutman, Phys. Rev. Lett. 121, 044801 (2018).

[5] J. Močkus, Optimization Techniques IFIP Technical Conference, Novosibirsk (1975), p. 400.

[6] B. Shahriari, K. Swersky, Z. Wang, R. P. Adams, and N. de Freitas, Proc. IEEE 104, 148 (2016).

[7] E. Brochu, V. M. Cora, and N. de Freitas, Univ. of British Columbia Tech. Rep. No. UBC TR-2009-023 and arXiv: 1012.2599, 2010.

[8] C. E. Rasmussen and C. K. I. Williams, Gaussian Processes for Machine Learning (MIT Press, Cambridge, MA, 2006).

[9] P. I. Frazier and J. Wang, Bayesian optimization for materials design, in Information Science for Materials Discovery and Design, edited by T. Lookman, F. J. Alexander, and K. Rajan (Springer International Publishing, Cham, 2016) pp. 45-75.

[10] S. Sano, T. Kadowaki, K. Tsuda et al., J. Pharm. Innovation 15, 333 (2019).

[11] M. M. Noack, K. G. Yager, M. Fukuto, G. S. Doerk, R. Li, and J. A. Sethian, Sci. Rep. 9, 11809 (2019).

[12] V. T. Taasti, L. Hong, J. S. Shim, J. O. Deasy, and M. Zarepisheh, Med. Phys. 47, 3286 (2020).

[13] A. Ekström, C. Forssén, C. Dimitrakakis, D. Dubhashi, H. T. Johansson, A. S. Muhammad, H. Salomonsson, and A. Schliep, J. Phys. G 46, 095101 (2019).

[14] M. McIntire, D. Ratner, and S. Ermon, Proceedings of the Thirty-Second Conference on Uncertainty in Artificial Intelligence (UAI'16) (AUAI Press, Arlington, Virginia, USA, 2016), pp. 517-526.

[15] J. Kirschner, M. Mutný, N. Hiller, R. Ischebeck, and A. Krause, Proceedings of the 36th International Conference on Machine Learning (ICML) (2019), pp. 3429-3438, http://proceedings.mlr.press/v97/kirschner19a.html.

[16] J. Duris, D. Kennedy, A. Hanuka, J. Shtalenkova, A. Edelen, P. Baxevanis, A. Egger, T. Cope, M. McIntire, S. Ermon, and D. Ratner, Phys. Rev. Lett. 124, 124801 (2020).

[17] A. Hanuka, J. Duris, J. Shtalenkova, D. Kennedy, A. Edelen, D. Ratner, and X. Huang, Proceedings of the Machine Learning for the Physical Sciences Workshop, 
NeurIPS (2019), https://ml4physicalsciences.github.io/ 2019/files/NeurIPS_ML4PS_2019_85.pdf.

[18] R. J. Shalloo et al., Nat. Commun. 11 (2020).

[19] S. Jalas, M. Kirchen, P. Messner, P. Winkler, L. Hübner, J. Dirkwinkel, M. Schnepp, R. Lehe, and A. R. Maier, Phys. Rev. Lett. 126, 104801 (2021).

[20] R. Hettel, in 9th European Particle Accelerator Conference (EPAC 2004), Lucerne, Switzerlan (2004), https://www.slac .stanford.edu/cgi-bin/getdoc/slac-pub-11117.pdf.

[21] D. Duvenaud, J. Lloyd, R. Grosse, J. Tenenbaum, and G. Zoubin, Proceedings of the 30th International Conference on Machine Learning 28, 1166 (2013), http://proceedings .mlr.press/v28/duvenaud13.html.

[22] S. Sun, G. Zhang, C. Wang, W. Zeng, J. Li, and R. Grosse, Proceedings of the 35th International Conference on Machine Learning (2018), pp. 4828-4837, http:// proceedings.mlr.press/v80/sun18e.html.

[23] A. G. Wilson, Z. Hu, R. R. Salakhutdinov, and E. P. Xing, in Advances in Neural Information Processing Systems 29 (2016) pp. 2586-2594, https://proceedings.neurips.cc/paper/ 2016/file/bcc0d400288793e8bdcd7c19a8ac0c2b-Paper.pdf.

[24] R. Calandra, J. Peters, C. E. Rasmussen, and M. P. Deisenroth, Proceedings of the 2016 International Joint Conference on Neural Networks (IJCNN) (2016), pp. 33383345, https://doi.org/10.1109/IJCNN.2016.7727626.

[25] A. C. Damianou and N. D. Lawrence, Proceedings of the 16th International Conference on Artificial Intelligence and Statistics (AISTATS) 31, 207 (2013).

[26] X. Yang, D. Barajas-Solano, G. Tartakovsky, and A. M. Tartakovsky, J. Comput. Phys. 395, 410 (2019).

[27] G. Camps-Valls, L. Martino, D. H. Svendsen, M. CamposTaberner, J. Muõoz-Marí, V. Laparra, D. Luengo, and F. J. García-Haro, Applied Soft Computing 68, 69 (2018).

[28] A. M. Tartakovsky and R. Tipireddy, 52nd Hawaii International Conference on System Sciences HICSS 2019 (2019), p. 1, https://scholarspace.manoa.hawaii.edu/ handle/10125/59779.

[29] J.-L. Wu, C. Michelén-ströfer, and H. Xiao, Comput. Fluids, 193, 104292 (2019).

[30] E. M. Constantinescu and M. Anitescu, Int. J. Uncertainty Quantification 3, 47 (2013).
[31] D. MacKay, NATO ASI series F Computer and Systems Sciences 168, 133 (1998), http://www.inference.org.uk/ mackay/gpB.pdf.

[32] R. Neal, Bayesian Learning for Neural Networks, Lecture Notes in Statistics (Springer, New York, 2012).

[33] N. Wiener, Acta Math. 55, 117 (1930).

[34] F. Aiolli, JMLR: Workshop and Conference Proceedings 27, 81 (2012), http://proceedings.mlr.press/v27/aiolli12a/ aiolli12a.pdf.

[35] W.-C. Chang, Y. Wu, H. Liu, and Y. Yang, 31st AAAI Conference on Artificial Intelligence, AAAI 2017 (2017), p. 1763.

[36] M. U. Gutmann and J. Corander, J. Mach. Learn. Res. 17, 1 (2016), https://jmlr.org/papers/volume17/15-017/15-017.pdf.

[37] E. Meeds and M. Welling, Uncertainty in Artificial Intelligence-Proceedings of the 30th Conference, UAI 2014 (2014), p. 593, arXiv:1401.2838.

[38] R. C. Conant and W. Ross Ashby, Int. J. Syst. Sci. 1, 89 (1970).

[39] R. Roussel, A. Hanuka, and A. Edelen, Multi-objective bayesian optimization for accelerator tuning, arXiv: 2010.09824.

[40] X. Huang, Beam-based Correction and Optimization for Accelerators (CRC Press, Taylor \& Francis Group, Boca Raton, FL, 2019).

[41] S. Tomin, G. Geloni, I. Agapov, I. Zagorodnov, Y. Fomin, Y. Krylov, A. Valintinov, W. Colocho, T. Cope, A. Egger, and D. Ratner, Proceedings of the 7th International Particle Accelerator Conference, Busan, Korea (2016).

[42] J. Safranek, Nucl. Instrum. Methods Phys. Res., Sect. A 388, 27 (1997).

[43] J. Degrave, M. Hermans, J. Dambre, and F. Wyffels, Front. Neurorobot. 13, 6 (2019).

[44] A. Edelen, N. Neveu, M. Frey, Y. Huber, C. Mayes, and A. Adelmann, Phys. Rev. Accel. Beams 23, 044601 (2020).

[45] F. Chollet et al., Keras, https://keras.io (2015).

[46] M. Abadi et al., Tensorflow: Large-scale machine learning on heterogeneous systems, (2015), https://www.tensorflow .org/.

[47] P. A. Brodtkorb and J. D'Errico, numdifftools, (2015), https://numdifftools.readthedocs.io/en/latest/index.html. 